\title{
Comparison of the Male Osteoporosis Risk Estimation Score (MORES) With FRAX in Identifying Men at Risk for Osteoporosis
}

\author{
Alvab R. Cass, $M D, S M^{1}$ \\ Angela J. Shepherd, $M D^{1}$ \\ Rechelle Asirot, $M D^{2}$ \\ Manju Mabajan, $M D^{3}$ \\ Maimoona Nizami, $M D^{4}$ \\ 'Department of Family Medicine, \\ The University of Texas Medical Branch, \\ Galveston, Texas \\ ${ }^{2}$ Peacehealth Medical Group, Eugene, \\ Oregon \\ ${ }^{3}$ Covenant Clinic, Oelwein, Iowa \\ ${ }^{4}$ Kingwood Medical Center, Kingwood, \\ Texas
}

\begin{abstract}
PURPOSE We wanted to compare the male osteoporosis risk estimation score (MORES) with the fracture risk assessment tool (FRAX) in screening men for osteoporosis.

METHODS This study reports analysis of data from the Third National Health and Nutrition Examination Survey (NHANES III), a nationally representative sample of the US population, comparing the operating characteristics of FRAX and MORES to identify men at risk for osteoporosis using a subset of 1,498 men, aged 50 years and older, with a valid dual-energy $x$-ray absorptiometry (DXA) scan. DXAderived bone mineral density using a T score of -2.5 or lower at either the femoral neck or total hip defined the diagnosis of osteoporosis. Outcomes included the operating characteristics, area under the receiver-operator characteristic curve, and agreement of the FRAX and MORES.
\end{abstract}

RESULTS Sixty-seven (4.5\%) of the 1,498 men had osteoporosis of the hip. The sensitivity, specificity, and area under the curve (AUC) for the MORES were 0.96 (95\% Cl, 0.87-0.99), 0.61 (95\% Cl, 0.58-0.63), and 0.87 (95\% Cl, 0.84-0.91), respectively. The sensitivity, specificity, and AUC for the FRAX were $0.39(95 \%$ $\mathrm{Cl}, 0.27-0.51), 0.89(95 \% \mathrm{Cl}, 0.88-0.91)$, and $0.79(95 \% \mathrm{Cl}, 0.75-0.84)$ respectively. Agreement was poor.

CONCLUSIONS Compared with the MORES, the FRAX underperformed as a screening strategy for osteoporosis using the threshold score suggested by the US Preventive Services Task Force (USPSTF). An integrated approach that uses the MORES to determine which men should have a DXA scan and the FRAX to guide treatment decisions, based on the risk of a future fracture, identified $82 \%$ of men who were candidates for treatments based on National Osteoporosis Foundation guidelines.

Ann Fam Med 2016;14:365-369. doi: 10.1370/afm.1945.

\section{INTRODUCTION}

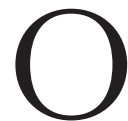
steoporosis leads to bone fragility and an increased risk of fractures. The World Health Organization (WHO) considers osteoporosis a critical health problem ${ }^{1}$ and a major cause of morbidity and mortality in the aging population. ${ }^{2,3}$ Men and women with osteoporosis experience hip fractures at a similar rate, ${ }^{4}$ but men experience a worse 1 -year mortality. ${ }^{5,6}$ Evidence indicates the benefits of bisphosphonate therapy in men are similar to those in women. ${ }^{7-10}$ Despite treatment benefits, screening recommendations for men lag behind those for women.

In 2011 the US Preventive Services Task Force (USPSTF) concluded, "The current evidence is insufficient to assess the balance of benefits and harms of screening for osteoporosis in men," but went on to state, "the men most likely to benefit from screening would have a 10 -year risk of an osteoporotic fracture equal to or greater than the risk of an osteoporotic fracture in 65 -year-old white women without additional risk factors for fracture," which equates to a risk of 9.3\%." The USPSTF correctly noted that the World Health Organization's fracture risk assessment tool 
$(\text { FRAX) })^{12,13}$ is designed to predict the 10-year risk of a hip fracture or a major osteoporotic fracture (hip, humerus, forearm, or spine) but failed to cite any references to support using the FRAX as a screening instrument for osteoporosis for men or women.

The purpose of this study is to compare the operating characteristics and level of agreement of the Male Osteoporosis Risk Estimation Score (MORES) ${ }^{14}$ and the FRAX ${ }^{12,13}$ in determining which men should be referred for a diagnostic dual-energy $\mathrm{x}$-ray absorptiometry (DXA) scan.

\section{METHODS}

We compared the MORES with the FRAX using a cross-sectional sample of men, representative of the US population, included in the National Health and Nutrition Examination Survey III (NHANES III). The MORES and FRAX outcomes were derived blinded to the results of the dual-energy x-ray absorptiometry (DXA) scans used to measure bone mineral density and the value of the alternate instrument.

When we developed the MORES, we randomly assigned the 2,995 men, aged 50 years and older, from the NHANES III data set to either a development or a validation cohort. ${ }^{14}$ For this study, we used the 1,498 men from the validation cohort. We defined osteoporosis based on the bone mineral density of the femoral neck or total hip using the non-Hispanic white female, aged 20 to 29 years, the NHANES III reference cohort. ${ }^{15}$ We included the bone mineral density of the total hip because it predicts future hip as well as femoral neck ${ }^{16}$ fractures and is used commonly in clinical practice.

The WHO, when it developed the FRAX, used this same reference cohort to define osteoporosis based on the bone mineral density of the femoral neck. ${ }^{12,13,17}$ By using both the femoral neck and total hip bone density measures, we were able to compare the MORES with the FRAX directly. Vertebral (spinal) bone density measurements were not available in NHANES III.

The NHANES III data set contains the necessary data to define or approximate the variables used in the MORES and the FRAX. The MORES and FRAX were used to classify men into to screen and do-not-screen categories.

\section{Instruments}

The MORES uses age, weight, and history of chronic obstructive pulmonary disease (COPD) to stratify the risk for osteoporosis in men aged 50 years and older.A score of 6 points or higher yielded a sensitivity of 0.93 , a specificity of 0.59 , and an area under the curve (AUC) of 0.832 .
The FRAX, a Web-based calculator, uses age, sex, race/ethnicity, body mass index (BMI), paternal history of hip fracture, personal history of a low-impact fracture, smoking, alcohol consumption, use of glucocorticoids, history of rheumatoid arthritis, and history of secondary causes of osteoporosis to predict risk of a hip fracture or a major osteoporotic fracture.

The NHANES III data set also includes bone mineral density results for the femoral neck and total hip for men aged 50 years and older. NHANES III bone mineral density results were transformed to $\mathrm{T}$ scores (scores of -2.5 or lower at either the femoral neck or total hip defined the diagnosis of osteoporosis) based the bone mineral density of the femoral neck and total hip from the reference cohort.

\section{Analysis}

We calculated the sensitivity and specificity for the MORES and FRAX from $2 \times 2$ contingency tables.

We computed the MORES according to the algorithm developed in the initial MORES study. Table 1 summarizes the scoring algorithm derived from the regression coefficients of the MORES logistic regression model. A score of 6 or more points defined the screen group; a score of less than 6 points defined the do-not-screen group.

We calculated the FRAX online by entering the required clinical data. A 10-year risk of a major osteoporotic fracture of $9.3 \%$ or greater, as suggested by the USPSTF, defined the screen group $;$ a score of less than 9.3\% defined the do-not-screen group. ${ }^{11}$

We used the range of observed values for both instruments to construct receiver operator characteristic curves and obtained estimates of the AUC for both instruments. ${ }^{18}$

We calculated 2 statistics from a $2 \times 2$ contingency table of paired responses for the MORES and FRAX. We used the McNemar $\chi^{2}$ statistic to test for the null

\section{Table 1. Male Osteoporosis Risk Estimation Score (MORES)}

\begin{tabular}{lc}
\hline Risk Factor & MORES Points ${ }^{\mathbf{a}}$ \\
\hline Age, $y$ & 0 \\
$\leq 55$ (reference) & 3 \\
$56-74$ & 4 \\
$\geq 75$ & \\
Weight, kg & 6 \\
$\leq 70$ & 4 \\
$71-80$ & 0 \\
$\geq 80$ (reference) & 3 \\
COPD & \\
\hline COPD = chronic obstructive pulmonary disease. \\
Screening threshold is 6 points or greater.
\end{tabular}


hypothesis of marginal homogeneity that the discordant cells contain equal proportions. ${ }^{19}$ We used the $\kappa$ statistic to test for the level of agreement, taking onto account the agreement occurring by chance. ${ }^{20}$

We estimated that $75(5 \%)$ men in the cohort of 1,498 men aged 50 years and older will have osteoporosis. This group determined the width of the 95\% confidence intervals around an estimate of the sensitivity of the MORES and FRAX. With a sample size of 75 , a 2 -sided $95 \% \mathrm{CI}$ for the sensitivity, using the large sample normal approximation, will extend 0.09 from the observed sensitivity, with an expected sensitivity of 0.80 , and 0.11 from the observed sensitivity, with an expected sensitivity of 0.50 .

A sample size of 1,462 pairs (1,498 expected) will have $90 \%$ power to detect a difference in proportions of discordant pairs of 0.05 when the proportion of discordant pairs is expected to be 0.35 and the method of analysis is a McNemar test of equality of paired proportions with an alpha of 0.05. Calculations were done using nQuery Advisor 6.0 (Statistical Solutions LTD).

The Institutional Review Board for Human Subjects Research granted approval for this study. All analyses used weighted data based on sampling of participants in the NHANES III data set.

\section{RESULTS}

The mean age of men in the sample population was $64.2 \pm 9.7$ years; $88.5 \%$ were non-Hispanic white, $8.5 \%$ were African-American, and $2.9 \%$ were MexicanAmerican. Sixty-seven men (4.5\%) had osteoporosis of the femoral neck and/or total hip. Table 2 summarizes the distribution of the risk factors from the MORES and FRAX.

The MORES and FRAX have different operating characteristics for identifying men with osteoporosis (Table 3). Based on the MORES, 41.8\% $(n=626)$ of the men would be referred for DXA, of whom $10.2 \%$ $(n=64)$ had osteoporosis. In comparison, based on the FRAX, $12.3 \%(n=185)$ of the men would be referred for DXA, of whom $14.1 \%(\mathrm{n}=26)$ had osteoporosis. Compared with the FRAX, the MORES had greater sensitivity (0.96 vs $0.39)$ but had lower specificity (0.61 vs 0.89 ). Given the low prevalence of osteoporosis, neither instrument served as a rule-in test; however, both tools were reasonably effective as a rule-out test. The MORES had a higher AUC, which approached statistical significance (Figure 1).

The McNemar test was highly significant, $P<.001$, indicating nonequivalence of the tests. The $\kappa$ statistic was $0.16, P<.001$, which indicates a level of agreement barely more than chance.

\section{Table 2. Distribution of Variables Used in the FRAX and MORES}

\begin{tabular}{|c|c|}
\hline Variables & Value \\
\hline \multicolumn{2}{|l|}{ FRAX } \\
\hline Age, mean (SD), y & $64.2(9.7)$ \\
\hline \multicolumn{2}{|l|}{ Race/ethnicity } \\
\hline Non-Hispanic white, No. (\%) & $1,326(88.5)$ \\
\hline African-American, No. (\%) & $128(8.5)$ \\
\hline Mexican-American, No. (\%) & $44(2.9)$ \\
\hline Height, mean (SD), cm & $174.6(6.8$ \\
\hline Weight, mean (SD), kg & $82.9(14.6)$ \\
\hline BMI, mean (SD) & $27.2(4.3)$ \\
\hline History of low-impact fracture, \% & $11(0.7)$ \\
\hline Parental history of hip fracture, $\%^{a}$ & $117(7.8)$ \\
\hline Current smoker, \% & $418(27.9)$ \\
\hline Use of glucocorticoids, \% ${ }^{b}$ & $25(1.6)$ \\
\hline History of rheumatoid arthritis, \% & $57(3.8)$ \\
\hline Secondary causes of osteoporosis, \%c & $7(0.5)$ \\
\hline Current alcohol use: ${ }^{3} 3$ drinks per day, $\%$ & $73(4.9)$ \\
\hline \multicolumn{2}{|l|}{ MORES } \\
\hline \multicolumn{2}{|l|}{ Age, y } \\
\hline$\leq 55, \%$ & $337(22.5)$ \\
\hline $56-74, \%$ & $907(60.6)$ \\
\hline$\geq 75, \%$ & $254(17.0)$ \\
\hline \multicolumn{2}{|l|}{ Weight, kg } \\
\hline$\leq 70, \%$ & $268(17.9)$ \\
\hline $71-80, \%$ & $386(25.8)$ \\
\hline$>80, \%$ & $843(56.3)$ \\
\hline History of COPD, \% & $150(10.0)$ \\
\hline \multicolumn{2}{|l|}{ Bone mineral density ${ }^{d}$} \\
\hline Osteoporosis, \% & $67(4.5)$ \\
\hline Osteopenia, \% & $501(33.4)$ \\
\hline Normal, \% & $930(62.1)$ \\
\hline
\end{tabular}

$\mathrm{BMI}=$ body mass index; $\mathrm{COPD}=$ chronic obstructive pulmonary disease; FRAX = a fracture risk assessment tool; MORES = Male Osteoporosis Risk Estimation Score; NHANES III = National Health and Nutrition Examination Survey III.

a NHANES III included only maternal history of fracture.

b NHANES III included only current use of glucocorticoids.

c NHANES III included data to support only type 1 diabetes, hypogonadism, and hyperthyroidism.

${ }^{d}$ Classified based on bone mineral density of total hip and/or femoral neck.

Table 3. A Comparison of the Operating Characteristics of the FRAX and MORES for Predicting Osteoporosis in Men Based on T Scores From the Hip

\begin{tabular}{lcc}
\hline Parameter & FRAX & MORES \\
\hline Sensitivity $(95 \% \mathrm{CI})$ & $0.39(0.27-0.51)$ & $0.96(0.87-0.99)$ \\
Specificity $(95 \% \mathrm{Cl})$ & $0.89(0.87-0.91)$ & $0.61(0.58-0.63)$ \\
Predictive value positive (95\% CI) & $0.14(0.09-0.20)$ & $0.10(0.08-0.13)$ \\
Predictive value negative (95\% CI) & $0.97(0.96-0.98)$ & $1.00(0.99-1.00)$ \\
Area under curve (95\% CI) & $0.79(0.74-0.84)$ & $0.87(0.84-0.91)$ \\
\hline FRAX = a fracture risk assessment tool; MORES = Male Osteoporosis Risk Estimation Score. \\
\hline
\end{tabular}




\section{Figure 1. Comparison of the areas under the receiver operating characteristic curves of the MORES and FRAX for predicting osteoporosis in men based on T scores from the hip.}

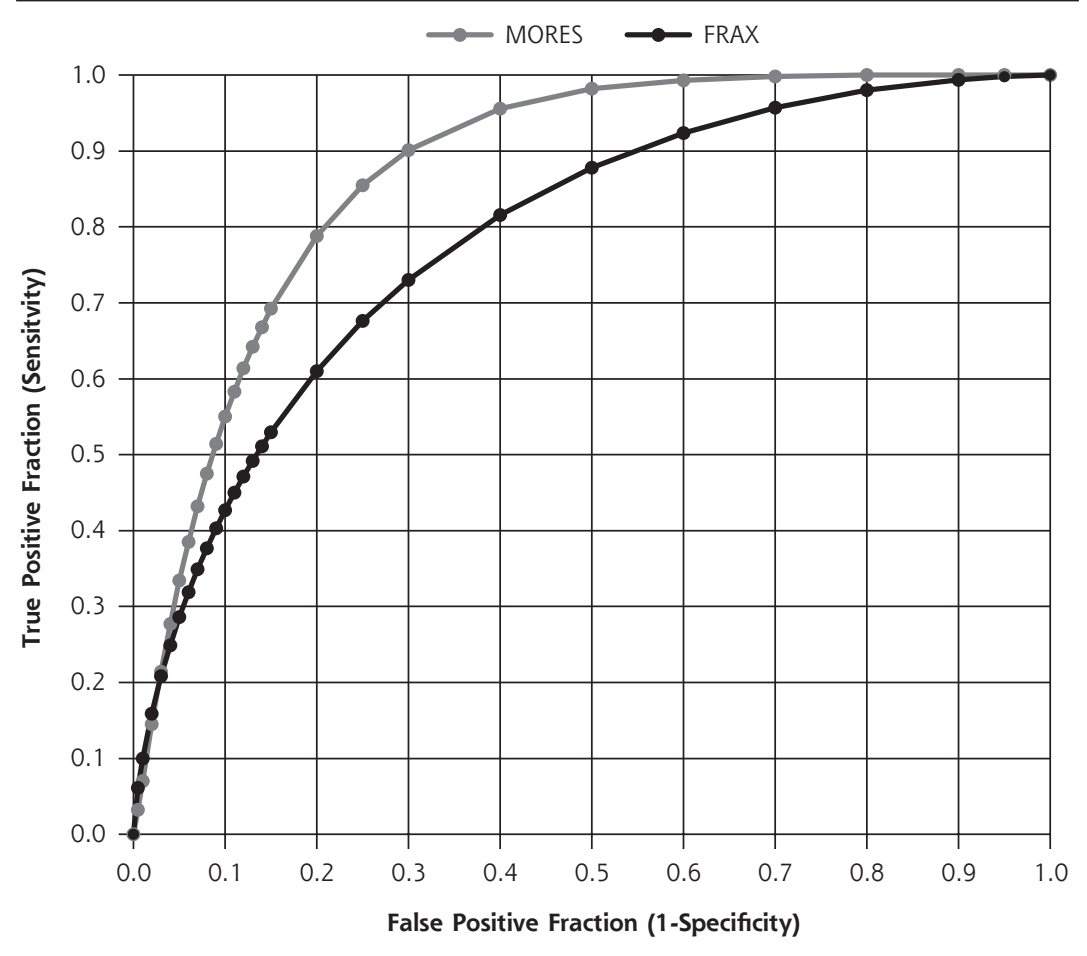

$\mathrm{AUC}=$ area under the curve; $\mathrm{FRAX}=\mathrm{a}$ fracture risk assessment tool; $\mathrm{MORES}=$ Male Osteoporosis Risk Estimation Score.

\section{DISCUSSION}

The 2011 USPSTF osteoporosis screening guidelines made no recommendation for or against screening men, leaving the decision to clinicians and the men for whom they provide care. By comparing the FRAX and the MORES as screening tools to aide clinicians in identifying men at risk for osteoporosis, we found the MORES is more effective based on the significantly higher sensitivity.

The MORES incorporates age and weight, 2 major risk factors for osteoporosis, and a history of COPD, to predict men at increased risk of osteoporosis. The FRAX uses a complex set of risk factors to predict future fractures. In the NHANES III data set, the FRAX did not perform well as a screening test for osteoporosis at the suggested threshold. The FRAX may indirectly predict osteoporosis given the association of osteoporosis and fragility fractures and by sharing age and weight as risk factors with the MORES.

The strengths of this study include use of a sample of men representative of the US population, and that it is the first study we found that evaluated the USPSTF's reliance on the FRAX to identify men at risk for osteoporosis. ${ }^{11}$ The NHANES III data set also had some limitations in that it derives from data from 1988 to 1994. The distribution of risk factors associated with osteoporosis and risk of fracture may have changed with time, but the impact of these factors on osteoporosis and fracture risk is likely unaffected. The data set did not allow for perfect modeling of the FRAX variables, ie, the data did not contain information for paternal hip fracture. This study did not address vertebral osteoporosis, because data are lacking in NHANES III, and vertebral osteoporosis is not used to calculate the FRAX. The MORES, however, does identify men at risk of vertebral osteoporosis. ${ }^{21}$

By 2030, osteoporotic fractures and the resultant functional impairment is expected to increase at a greater rate in men than in women. ${ }^{22-27}$ For clinicians and patients who are concerned about osteoporosis, the MORES provides a simple clinical approach to identifying men at greatest risk for osteoporosis and for whom a DXA scan is reasonable. The MORES can be readily administered at the time of an office encounter. The FRAX requires a computer program and clinical information less easily obtained, but electronic health records can simplify access through Internet links.

An integrated approach that uses the MORES to identify men who should have a diagnostic DXA scan and then uses the FRAX to guide treatment decisions may be the optimal approach. For example, 178 (11.9\%) men in the sample were candidates for treatment based on the National Osteoporosis Foundation guidelines. ${ }^{28}$ Sixty-seven men had osteoporosis and 111 men had osteopenia of the hip associated with a 10 -year risk greater than $3 \%$ for a hip fracture or greater than $20 \%$ for a major osteoporotic fracture. Integrating the MORES and FRAX identified $82 \%(n=145)$ of the men who were candidates for treatment. In contrast, the FRAX, as the sole approach, identified 50\% $(\mathrm{n}=89)$ of candidates for treatment. Clinicians who refer men for DXA testing based on the MORES could use the bone mineral density results to calculate the FRAX and engage in a shared decision about treatment during a follow-up encounter. 
We suggest that the USPSTF's reliance on the FRAX to identify men who could be screened for osteoporosis is an application for which FRAX was not designed. We believe the MORES is a better screening tool for osteoporosis in men than the FRAX, whereas the FRAX is a valuable tool to guide treatment decisions.

To read or post commentaries in response to this article, see it online at http://www.annfammed.org/content/14/4/365.

Key words: osteoporosis; mass screening; sensitivity and specificity

Submitted September 1, 2015; submitted, revised, February 4, 2016; accepted February 22, 2016.

Funding support: Funded in part by the Texas Academy of Family Physicians Foundation grant "A Comparative Analysis of the FRAX and the Male Osteoporosis Risk Estimation Score."

Previous presentations: Our findings were presented at the $41^{\text {st }}$ Annual North American Primary Care Research Group Meeting, November 9-13, 2013; Ottawa, Ontario; and the Annual Scientific Meeting of the American Geriatrics Society; May 15-18, 2014; Orlando, Florida.

\section{References}

1. International Osteoporosis Foundation. Osteoporosis in the European Community: A Call for Action. Nyon, Switzerland: International Osteoporosis Foundation; 2002.

2. Healthy People 2010. Arthritis, osteoporosis, and chronic back conditions. https://www.healthypeople.gov/2020/topics-objectives/ topic/Arthritis-Osteoporosis-and-Chronic-Back-Conditions. Published 2000. Accessed Jan 18, 2008.

3. Kanis JA, McCloskey EV. Evaluation of the risk of hip fracture. Bone. 1996;18(3)(Suppl):127S-132S.

4. Kanis JA, Oden A, Johnell O, Jonsson B, de Laet C, Dawson A. The burden of osteoporotic fractures: a method for setting intervention thresholds. Osteoporos Int. 2001;12(5):417-427.

5. Center JR, Nguyen TV, Schneider D, Sambrook PN, Eisman JA. Mortality after all major types of osteoporotic fracture in men and women: an observational study. Lancet. 1999;353(9156):878-882.

6. Forsén L, Sogaard AJ, Meyer HE, Edna T, Kopjar B. Survival after hip fracture: short- and long-term excess mortality according to age and gender. Osteoporos Int. 1999;10(1):73-78.

7. Orwoll $E$, Ettinger $M$, Weiss $S$, et al. Alendronate for the treatment of osteoporosis in men. N Engl J Med. 2000;343(9):604-610.

8. Gonnelli S, Cepollaro C, Montagnani A, et al. Alendronate treatment in men with primary osteoporosis: a three-year longitudinal study. Calcif Tissue Int. 2003;73(2):133-139.

9. Sato Y, Iwamoto J, Kanoko T, Satoh K. Risedronate sodium therapy for prevention of hip fracture in men 65 years or older after stroke. Arch Intern Med. 2005;165(15):1743-1748.

10. Sawka AM, Papaioannou A, Adachi JD, Gafni A, Hanley DA, Thabane $\mathrm{L}$. Does alendronate reduce the risk of fracture in men? $A$ meta-analysis incorporating prior knowledge of anti-fracture efficacy in women. BMC Musculoskelet Disord. 2005;6:39.
11. U.S. Preventive Services Task Force. Screening for osteoporosis: U.S. preventive services task force recommendation statement. Ann Intern Med. 2011;154(5):356-364

12. Kanis JA, Johnell O, Oden A, Johansson H, McCloskey E. FRAX and the assessment of fracture probability in men and women from the UK. Osteoporos Int. 2008;19(4):385-397.

13. World Health Organization Collaborating Centre for Metabolic Bone Diseases. Welcome to FRAX ${ }^{\circledast}$. http://www.shef.ac.uk/FRAXI. Accessed Jan 24, 2012.

14. Shepherd AJ, Cass AR, Carlson CA, Ray L. Development and internal validation of the male osteoporosis risk estimation score. Ann Fam Med. 2007;5(6):540-546.

15. Looker AC, Wahner HW, Dunn WL, et al. Updated data on proximal femur bone mineral levels of US adults. Osteoporos Int. 1998;8(5):468-489.

16. Stone $K L$, Seeley DG, Lui LY, et al. BMD at multiple sites and risk of fracture of multiple types: long-term results from the study of osteoporotic fractures. J Bone Miner Res. 2003;18(11):1947-1954.

17. National Center for Health Statistics. National health and nutrition examination survey. http://www.cdc.gov/nchs/nhanes. Published 2002.

18. Eng J. ROC analysis: web-based calculator for ROC curves. http:// www.jrocfit.org. Published 2014. Accessed Jun 30, 2015.

19. MCNEMAR Q. Note on the sampling error of the difference between correlated proportions or percentages. Psychometrika. 1947;12(2):153-157.

20. Cohen J. A coefficient of agreement for nominal scales. Educ Psychol Meas. 1960;20(1):37-46.

21. Shepherd AJ, Cass AR, Ray L. Determining risk of vertebral osteoporosis in men: validation of the male osteoporosis risk estimation score. J Am Board Fam Med. 2010;23(2):186-194.

22. Melton LJ III, O'Fallon WM, Riggs BL. Secular trends in the incidence of hip fractures. Calcif Tissue Int. 1987;41(2):57-64.

23. Office of Technology Assessment. Hip Fracture Outcomes in People Age 50 and Over, OTA-BP-H-120. Washington, DC: US Government Printing Office; 1994.

24. Riggs BL, Melton LJ III. The worldwide problem of osteoporosis: insights afforded by epidemiology. Bone. 1995;17(5)(Suppl):505S-511S.

25. Bacon WE. Secular trends in hip fracture occurrence and survival: age and sex differences. J Aging Health. 1996;8(4):538-553.

26. Kanis J, Johnell O, Gullberg B, et al. Risk factors for hip fracture in men from southern Europe: the MEDOS study. Mediterranean Osteoporosis Study. Osteoporos Int. 1999;9(1):45-54.

26. US Census Bureau. US Census Bureau: Projection of the Total Resident Population by 5-year Age Groups. Washington, DC: US Government Printing Office; 2001.

28. Cosman F, de Beur SJ, LeBoff MS, et al; National Osteoporosis Foundation. Clinician's guide to prevention and treatment of osteoporosis. Osteoporos Int. 2014;25(10):2359-2381. 